\title{
Comparing the efficacy and safety of IL-17 inhibitors for treatment of moderate-to-severe psoriasis: a randomized double blind pilot study with a review of literature
}

\author{
Nawaf AlMutairi', Bayoumy Ibrahim Eassa² \\ ${ }^{1}$ Department of Medicine, Faculty of Medicine, Kuwait University, Jabriya, Kuwait \\ ${ }^{2}$ Department of Dermatology, Farwaniya Hospital, Kuwait
}

Adv Dermatol Allergol 2021; XXXVIII (2): 281-288

DOI: https://doi.org/10.5114/ada.2019.91496

\begin{abstract}
Introduction: Psoriasis is a chronic skin disease in which interleukin-17A (IL-17A) has been found to play an important role. Commercially available anti-IL-17 drugs include brodalumab, ixekizumab (IXE), and secukinumab (SEC). Aim: To compare the safety and efficacy of IXE and SEC in patients with moderate-to-severe plaque psoriasis.

Material and methods: The patients were randomized to the IXE or SEC group. Effectiveness was estimated by Physician's Global Assessment (PGA), Psoriasis Area and Severity Index (PASI), and Dermatology Life Quality Index (DLQI). Safety was assessed by documentation of adverse effects (AEs), routine laboratory values, and injection-site and allergic reactions.

Results: There were 155 patients in the IXE group and 158 in the SEC group. At week 12, PASI 75 was 76.77\% (IXE) vs. $67.09 \%$ (SEC); PASI $9042.58 \%$ (IXE) vs. $32.28 \%$ (SEC); PGA score of 0 or 1 at week 40 (79.52\% vs. $74.4 \%)$ and at week $52(61.83 \%$ vs. 58.12\%) $(p<0.001)$. Also, DLQI score improvement was more pronounced in the IXE group. The rates and types of AEs were similar in both groups.

Conclusions: Although both groups demonstrated a robust clinical response, a significant improvement in patient quality of life, and a satisfactory safety profile, the IXE group scored a notch higher.
\end{abstract}

Key words: plaque psoriasis, IL-17 inhibitors, secukinumab, ixekizumab.

\section{Introduction}

Psoriasis is a chronic disease characterized by T-cell mediated systemic inflammatory response [1, 2]. According to various reports, approximately $2 \%$ of the world's population is affected by psoriasis [3, 4]. Psoriasis is also associated with markedly reduced quality of life and multiple co-morbidities, with the metabolic syndrome and cardiovascular disease being most significant [5, 6].

Conventionally, systemic agents like methotrexate, cyclosporin and acitretin have been used for the treatment of moderate-to-severe psoriasis [6]. However, they are associated with significant adverse effects and/or decreased efficacy. With the recent advancement in our knowledge at a molecular level, there has been a better understanding of the pathogenesis of psoriasis, which in turn has led to the development of several highly effective biological agents, thus reforming the treatment of psoriasis and fulfilling the needs of psoriasis patients.

The success of tumour necrosis factor- $\alpha$ (TNF- $\alpha$ ) inhibitors in the management of psoriasis has been linked to the suppression of the IL-23/IL-17 axis [7], which has been realized recently, due to the improved understanding of the pathogenesis of psoriasis, including the central role played by IL-17. IL-17, which secreting lymphocytes, including Th17 cells and others, plays a vital role in the regulation of the adaptive and innate immune systems as well as in the pathogenesis of psoriasis [8]. The proinflammatory cytokines belonging to the IL-17 family, including IL-17A, IL-17B, IL-17C, IL-17D, IL-17E, and IL-17F are the primary cytokines produced by Th17 cells [9-11].

Address for correspondence: Prof. Nawaf AlMutairi, Department of Medicine, Faculty of Medicine, Kuwait University, Post Box No. 280, Farwaniya 80000, Kuwait, phone: 0965-99370203, fax: 0965-24808167, e-mail: nalmut@usa.net Received: 18.10.2019, accepted: 18.11.2019. 
Of these, IL-17A has a major role to play in the immunologic defence against extracellular pathogens, as well as the pathogenesis of psoriasis and other immune-mediated diseases [11], which is mediated by several important functions of IL-17 including: recruitment of neutrophils, induction of a $\mathrm{Th}_{2}$ response, stimulation of macrophages to produce IL-1 $\beta$ and TNF- $\alpha$, and induction of matrix metalloproteinases (MMPs) [11].

With the increasing importance of IL-17 highlighted recently, the focus now has shifted to the development of newer biologic agents to target these cytokines $[9,12,13]$. Drugs targeting IL-17 currently available commercially include brodalumab, ixekizumab (IXE), and secukinumab (SEC). IXE and SEC both neutralize IL-7A; brodalumab acts on the IL-17A receptor, thus inhibiting the IL-17 pathway [14].

Head-to-head randomized trials are the best way to compare the efficacy and safety of these agents. Several studies in the past compared IL-17 inhibitors with other class of biologics. However, till this day, there has been no head-to-head clinical trial between the IL-17 antagonists.

\section{Aim}

Thus, we conducted this pilot study to compare the long-term safety and efficacy of treatment with IXE and SEC in patients with moderate-to-severe plaque psoriasis. Brodalumab is not available locally, so it could not be included in our comparative evaluation study.

\section{Material and methods}

This randomized, double-blind study was conducted at dermatology clinics in 5 dermatology centres associated with Farwaniya Hospital, State of Kuwait, beginning January 2017 through April 2019. Inclusion criteria were: patients 18 years of age or older, who had moderate-tosevere plaque psoriasis for at least 6 months. Moderateto-severe plaque psoriasis was defined by one or more of the following three criteria: $10 \%$ or more of the whole body-surface area involved, a score of 3 or higher on the Physician's Global Assessment (PGA; where the scores range from 0 to 5 , with $0=$ cleared; $1=$ minimal; 2 = mild; 3 = moderate; 4 = marked and $5=$ severe disease), and a score of 12 or more on the PASI (scores range from 0 to 72). Exclusion criteria were: the presence of nonplaque psoriasis, a recent serious systemic or local infection necessitating hospitalization or antibiotic therapy, use of conventional systemic drugs or phototherapy within the previous 4 weeks, or topical therapy within 2 weeks before randomization, or biologic therapy within the last 3 months before enrolment into the study. All patients provided written informed consent. The study was approved by the local Ethics Committee of the hospital, and was conducted in full compliance with the Declaration of Helsinki Guidelines.

This 52-week study comprised of two parts: initial 40 weeks of the treatment period, followed by 12 -week follow-up period. The treatment period was divided into 2 phases: week 0 to 12 as the induction phase, and week 13 to week 40 as the maintenance phase. After getting all routine baseline investigations, the patients were randomly assigned to receive either IXE (160 mg IXE) at weeks 0 , then $80 \mathrm{mg}$ IXE q2wks at weeks 2, 4, 6, 8, 10 , and 12 , and then $80 \mathrm{mg} \mathrm{SC} \mathrm{q4wks),} \mathrm{or} \mathrm{SEC} \mathrm{(300} \mathrm{mg}$ SEC with initial dosing at weeks 0, 1, 2, 3 and 4, followed by monthly maintenance dosing). In order to maintain the double blind nature of the study, during the first 12 weeks, each patient in both the groups, received weekly injections. Wherein, a placebo injection was given in the week when the patient was not scheduled to receive the assigned drug according to the recommended dosage schedule (thus, in the IXE group, placebo injections were given at weeks 1, 3, 5, 7, 9, and week 11. Whereas, in the SEC group, placebo injections were given at weeks 5 , $6,7,9,10,11)$. Thereafter, each patient in both the groups was given an injection once in 4 weeks (as per the recommended dosage schedule of both the drugs). Thus, effectively each patient in both the groups received once a week injection during the initial 12 weeks (Induction phase), and thereafter once every 4 weeks till week 40 (Maintenance phase).

Efficacy of the treatment was measured by PGA, the Psoriasis Area and Severity Index (PASI), and the Dermatology Life Quality Index (DLQI; on which scores range from 0 to 30, with higher scores indicating a more negative effect on quality of life). Whereas, safety and tolerability of the drug was assessed by documentation of AEs, routine hematologic and laboratory tests, and injectionsite and allergic reactions.

The primary end points of this study were the percentage of patients with PASI 75, and a score on the PGA of 0 or 1 at week 12 .

The secondary end points at week 12 included the percentage of patients achieving PASI 90 and the change in the DLQI score from baseline, which measured the effect of dermatologic diseases on the quality of life (on a scale from 0 to 30, with lesser scores indicating a minor effect on health-related quality of life). The DLQI evaluations included the percentage of patients with a DLQI score of 0 or 1 (indicating no effect of psoriasis), the percentage of patients with a decrease of at least 5 points (indicating a clinically noteworthy reduction), and mean reductions in DLQI scores from baseline. Treatmentemergent AEs were assessed throughout the 52 weeks of the study.

For the statistical analysis, a two-sided CochranMantel-Haenszel $\chi^{2}$ test was used. All tests were twosided. Multiplicity adjustment was performed only for the comparison of the primary end points between the 
two groups. If a given comparison in the test sequence was not significant, the remaining subsequent comparisons were also considered insignificant.

The primary end point and other critical end points linked with PGA and PASI scores at week 12 were analysed. Patients who were removed from the study because of unsatisfactory response or due to use of any treatment that was prohibited in the protocol, were considered in the analysis of end points as not having had a response and were given a score of zero in the analysis of continuous end points (e.g., variation in DLQI score) from that point onward. Patients with missing PGA or PASI scores at week 12 were considered as not having had a response. Variation in response rates and the corresponding $95 \%$ confidence intervals were calculated between the two groups, at week 12, and then again at week 52.

Safety evaluations included all the patients who received at least one dose of a study drug. The AEs were summarized separately for the two treatment phases (week 0 to week 12, and from week 13 to week 52). Differences between the two treatment groups in the change from baseline in scores on the PASI and DLQI and in other continuous variables were analysed by analysis of covariance, with the baseline value in the model. Wilcoxon two-sample tests were used to compare the betweengroup difference in the change.

\section{Results}

Out of the total of 463 patients that were screened for enrolment, 313 were found to be eligible for inclusion in the study. Those included were randomly assigned to one of two treatment groups in approximately $1: 1$ ratio. There were 155 patients in the IXE group, and 158 patients in the SEC group. Baseline demographic and disease characteristics of the patients were almost similar among the two treatment groups (Table 1). The mean age was approximately 42 years, and males constituted $72.84 \%$ of the total patients. The average duration of the disease was approximately 17 years. The extent of involvement as measured by BSA showed the mean body-
Table 1. Baseline demographic and clinical characteristics

\begin{tabular}{lcc}
\hline Characteristic & $\begin{array}{c}\text { Ixekizumab } \\
(n=155)\end{array}$ & $\begin{array}{c}\text { Secukinumab } \\
(n=158)\end{array}$ \\
\hline Age [years] & $42.0 \pm 11.3$ & $41.1 \pm 13.7$ \\
\hline Male sex, $n(\%)$ & $117(75.50)$ & $111(70.25)$ \\
\hline Weight $[\mathrm{kg}]$ & $87.3 \pm 15.4$ & $85.7 \pm 17.3$ \\
\hline Duration of psoriasis [years] & $18.1 \pm 18.7$ & $17.6 \pm 14.5$ \\
\hline $\begin{array}{l}\text { Body-surface area affected } \\
\text { by psoriasis (\%) }\end{array}$ & $27.3 \pm 13.2$ & $27.1 \pm 15.1$ \\
\hline
\end{tabular}

\begin{tabular}{lcc}
\hline PASI score & $17.6 \pm 8.4$ & $16.9 \pm 7.7$ \\
\hline PGA score, $n$ (\%): & 0 & 0 \\
\hline $0-2$ & $71(45.81)$ & $79(50.00)$ \\
\hline 3 & $62(40.00)$ & $68(43.04)$ \\
\hline 4 & $13(8.39)$ & $11(6.96)$ \\
\hline 5 & $12.3 \pm 7.2$ & $13.1 \pm 4.6$ \\
\hline DLQI score & & \\
\hline Tovious psoriasis treatment: & $155(100.0)$ & $158(100.0)$ \\
\hline Phototherapy & $87(56.13)$ & $91(57.60)$ \\
\hline Systemic non-biologic treatment & $64(41.29)$ & $69(43.67)$ \\
\hline Systemic biologic treatment & $28(18.06)$ & $26(16.46)$ \\
\hline
\end{tabular}

surface area affected by psoriasis to be approximately $27 \%$, and the mean baseline PASI score approximately 26. All the patients had used topical agents, and around $17 \%$ had even used biologic agents in their past treatments (Table 1).

\section{Effectiveness}

At week $12,67.09 \%$ of patients in the SEC group had at least $75 \%$ improvement in the PASI score, as compared with $76.77 \%$ of patients in the IXE group $(p<0.001)$ (Table 2). The percentage of patients achiev-

Table 2. Clinical responses at week 12

\begin{tabular}{lccc}
\hline Response & Ixekizumab $(n=155)$ & Secukinumab $(n=158)$ & $P$-value \\
\hline Improvement in PASI score: & & & $<0.001$ \\
\hline At least $75 \%, n(\%),(95 \% \mathrm{Cl})$ & $119(76.77),(8.1-18.6)$ & $106(67.09),(3.9-16.4)$ & $51(32.28),(6.2-21.3)$ \\
\hline At least $90 \%, n(\%),(95 \% \mathrm{Cl})$ & $66(42.58),(13.4-27.2)$ & $102(64.56),(6.8-23.7)$ & $<0.001$ \\
\hline PGA 0 or 1, $n(\%),(95 \% \mathrm{Cl})$ & $108(69.68),(13.9-27.4)$ & $89(56.33),(3.1-12.5)$ & $<0.001$ \\
\hline DLQI 0/1, $n(\%),(95 \% \mathrm{Cl})$ & $114(73.55),(10.8-22.9)$ & 0.1 \\
\hline
\end{tabular}

P-values are given for the comparison of rates between the Ixekizumab group and the Secukinumab group. CI - confidence interval, PGA - physician's global assessment, PASI - psoriasis area-and-severity index. 

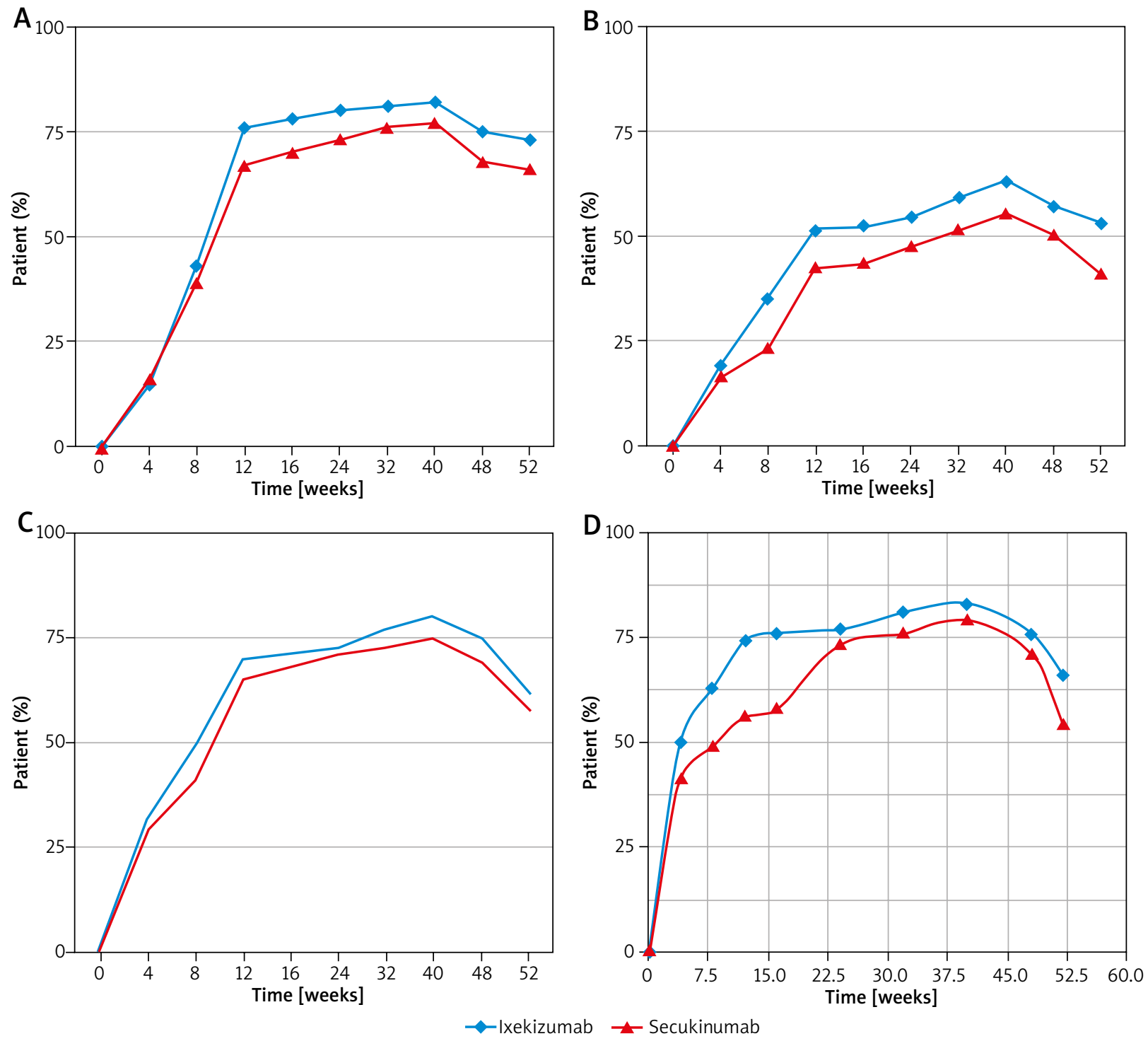

Figure 1. A - PASI 75, B - PASI 90, C - Physician's Global Assessment score (PGA), D - reduction in the DLQI score

ing PASI 75 was significantly greater in the IXE group than in the SEC group by week 12 , and at all time points through week 52 (Figure 1 A). The median time taken to achieve PASI 75 was 56 days in the IXE group, and 71 days in the SEC group $(p<0.001)$.

Also, a significantly higher (42.8\%) number of patients in the IXE group attained PASI 90 at week 12 than patients who received SEC (32.28\%) $(p<0.001)$ (Table 2). The onset of a clinical response was more rapid in patients treated with IXE, than in patients treated with SEC (Figure 1 B). The percentage of patients achieving PASI 90 increased over time in both the treatment groups, but was found to be significantly greater in the IXE group at all time points.

The proportion of patients who were clear of disease (a score of 0) or had minimal disease (a score of 1) based on the PGA at week 12 was higher in the IXE group (69.68\%), as compared with $64.56 \%$ in the SEC group ( $p<0.001)$ (Table 2). Also, a higher number of patients in the IXE group had the PGA score of 0 or 1 at week 40 (79.52\% vs. $74.4 \%)$ and at week 52 (61.83\% vs. 58.12\%) $(p<0.001)$ (Figure 1C). The median time taken to achieve a score of 0 or 1 on the PGA was 69 days in the IXE group, as compared to 77 days in the SEC group $(p<0.001)$.

Similarly, reduction in the DLQI score was also seen in a significantly higher number of patients in the IXE group than the SEC group through weeks 12 (73.55\% vs. 56.33\%, respectively) (Figure $1 \mathrm{D}$ ). Mean reductions in DLQI scores from baseline were greater in the IXE group than in the SEC group at all the time points assessed. Even during the follow-up period, more patients in the IXE group had 
a reduction in the DLQI score (DLQI at week 52: 66.33\% vs. $54.26 \%, p<0.001)$.

\section{Safety}

The most common AEs noticed in our study are summarized in Table 3. During the first 12 weeks, the proportion of patients with at least one reported AE were almost similar in both the groups (70.32\% vs. 68.98\%), all these were mild and no patient discontinued treatment. Also, no severe AEs were reported in any patient from the two groups. The most common AEs were nasopharyngitis, upper respiratory tract infection, headache and GIT abnormalities (nausea, abdominal pain and diarrhoea). Even at week 52, the rate and type of common adverse effects were similar in both groups (Table 3). No serious lifethreatening AE was reported, and none of the patients required hospitalization. Also, no patient discontinued treatment or had to reduce the drug dose due to any adverse effect. No noticeable changes in the physical examination were observed at any time during 52 weeks in both groups.

The proportion of subjects experiencing at least one AE was $71.61 \%$ in the IXE group and $69.62 \%$ in the SEC group. Mild to moderate infections and infestations were the most frequent adverse effects (58.71\% for IXE and $53.16 \%$ for SEC). Serious infections occurred in 3 (1.89\%) patients in the SEC group, and 5 (3.23\%) patients in the IXE group. No cutaneous malignancy or other malignant condition was reported. Major adverse cardiovascular events (myocardial infarction) were reported in $3 \mathrm{pa}$ tients; 1 from the SEC group, and 2 from the IXE group.

The proportion of patients with abnormalities in hematologic and laboratory measures, including tests of liver, were similar among the two groups (Table 3).

\section{Discussion}

The efficacy of biologic therapy for the treatment of psoriasis has been proved beyond doubt. But, there is a need to compare the efficacy and safety of these biologic agents, for best therapeutic benefits of psoriasis patients.

Interleukin-17 secreting T lymphocytes, including Th17 cells and others, play a vital role in the regulation of the adaptive and innate immune systems as well as in the pathogenesis of psoriasis [8]. The pro-inflammatory

Table 3. Adverse effects according to treatment groups

\begin{tabular}{|c|c|c|c|c|}
\hline \multirow[t]{2}{*}{ Parameter } & \multicolumn{2}{|c|}{ Week 0 - week 12} & \multicolumn{2}{|c|}{ Week 13 - week 52} \\
\hline & $\begin{array}{c}\text { Ixekizumab } \\
n(\%)\end{array}$ & $\begin{array}{c}\text { Secukinumab } \\
n(\%)\end{array}$ & $\begin{array}{c}\text { Ixekizumab } \\
n(\%)\end{array}$ & $\begin{array}{c}\text { Secukinumab } \\
n(\%)\end{array}$ \\
\hline \multicolumn{5}{|l|}{ Common adverse reactions: } \\
\hline Mild to moderate infections & $44(28.39)$ & $39(24.68)$ & $47(30.32)$ & $45(28.85)$ \\
\hline Headache & $19(12.26)$ & $19(12.03)$ & $26(16.77)$ & $18(11.39)$ \\
\hline Nasopharyngitis - sinusitis & $11(7.09)$ & $15(9.49)$ & $33(21.29)$ & $23(14.56)$ \\
\hline Upper RTI - bronchitis & $7(4.52)$ & $10(6.33)$ & $29(18.71)$ & $19(12.03)$ \\
\hline Pruritus & $3(1.94)$ & $7(4.43)$ & $11(7.09)$ & $8(5.06)$ \\
\hline Fatigue & $6(3.87)$ & $8(5.06)$ & $8(5.16)$ & $5(3.16)$ \\
\hline Arthralgia - back pain & $11(7.09)$ & $6(3.80)$ & $13(8.39)$ & $12(7.59)$ \\
\hline Cough & $5(3.23)$ & $9(5.70)$ & $4(2.58)$ & $7(4.43)$ \\
\hline Gastroenteritis (diarrhoea, nausea, abdominal pain) & $14(9.03)$ & $7(4.43)$ & $5(3.23)$ & $8(5.06)$ \\
\hline \multicolumn{5}{|l|}{ Serious adverse reactions: } \\
\hline Infections & $1(0.65)$ & $1(0.63)$ & $4(2.58)$ & $2(1.27)$ \\
\hline Cardiac/vascular & $0(0.00)$ & $0(0.00)$ & $2(1.29)$ & $1(0.63)$ \\
\hline Renal and urinary & $0(0.00)$ & $0(0.00)$ & $1(0.66)$ & $0(0.00)$ \\
\hline Gastrointestinal & $1(0.66)$ & $1(0.63)$ & $2(1.29)$ & $2(1.27)$ \\
\hline Others & $0(0.0)$ & $2(1.27)$ & $6(3.87)$ & $7(4.43)$ \\
\hline \multicolumn{5}{|l|}{ Hematologic laboratory abnormalities: } \\
\hline Decreased neutrophils & $0(0.0 \%)$ & $0(0.0 \%)$ & $2(1.29)$ & $3(1.89)$ \\
\hline Decreased lymphocytes & $0(0.0 \%)$ & $0(0.0 \%)$ & $1(0.66)$ & $2(1.27)$ \\
\hline Elevated ALT/SGPT & $1(0.66)$ & $2(1.27)$ & $3(1.94)$ & $3(1.89)$ \\
\hline Elevated AST/SGOT & $0(0.0 \%)$ & $1(0.63)$ & $1(0.66)$ & $2(1.27)$ \\
\hline
\end{tabular}


cytokines belonging to the IL-17 family, including IL-17A, IL-17B, IL-17C, IL-17D, IL-17E, and IL-17F are the primary cytokines produced by Th17 cells [9, 11]. Of these, IL-17A has a major role to play in the immunologic defence against extracellular pathogens, as well as the pathogenesis of psoriasis and other immune-mediated diseases $[15,16]$. Out of the six known subtypes of IL-17, increased expression of IL-17A, IL-17C and IL-17F has been seen in the inflammatory pathway involved in pathogenesis of psoriasis.

Interleukin-17A is expressed by a subset of T-lymphocytes called Th17 cells, at inflammatory sites. It exists in two forms, either as a homodimer (A/A) or as a heterodimer (A/F) with IL-17F. Because of the widespread expression of IL-17A receptors, most of the cell types are able to respond to the local production of IL-17A. IL-17A maintains a pro-inflammatory state by stimulating the release of cytokines and chemokines designed to recruit and activate both neutrophils and memory T-cells to the site of injury or inflammation [17].

The role of interleukin (IL)-17 and Th17 cells in the pathogenesis of autoimmune disorders has been elucidated in various genetic studies and correlative expression data in diseased tissues [18]. In addition to Th17 cells, IL-17 is also produced by $\gamma \delta$ T-cells, neutrophils, and mast cells [19]. The substantial role played by the mast cells and neutrophils in comparison to Th17 cells, in the release of IL-17 has been detailed in a study done on human skin using immunofluorescence (IF), showing that there are more mast cells and neutrophils producing IL-17 in psoriatic plaques than Th17 cells [19].

Th17 cells are now implicated in many of the autoimmune inflammatory responses, which were once attributed to Th1 cells. And, there are various reports linking aberrant Th17 response and increased IL-17A production in a number of autoimmune diseases e.g. rheumatoid arthritis, psoriasis, psoriatic arthritis, and ankylosing spondylitis [20, 21].

Interleukin-17 also plays a key role in host defence against extracellular bacteria and fungi. It acts on keratinocytes to upregulate the expression of chemokines af fecting the recruitment of various pro-inflammatory cells like myeloid dendritic cells, Th17 cells, and neutrophils to the lesion site. IL-17A is also known to induce the production of antimicrobial peptides and pro-inflammatory cytokines, which in turn, may amplify and sustain the immune responses in the skin [22]. IL-17A has been shown to downregulate the expression of filaggrin, and can thus potentiate the disruption of the skin barrier [23].

Recently, it has been demonstrated that IL-17A is also expressed by many cells including mast cells, neutrophils, dendritic cells, $\gamma \delta$-T cells, macrophages and natural killer cells. Thus, the inhibition of IL-17A as a therapeutic target in autoimmune diseases could also lead to different physiological effects, other than the suppression of Th17 cell activity [18].
Recent developments in the understanding of the pathogenesis of psoriasis have identified interleukin (IL)-17 as the key proinflammatory cytokine, resulting in the discovery of many newer drugs that target this cytokine or its associated receptors [14]. The increased presence of IL-17A in psoriatic plaques stimulates the local keratinocytes to produce certain pro-inflammatory chemokines, which in turn induce recruitment of inflammatory cells to the site. Thus, IL-17A-mediated stimulation of macrophages, fibroblasts and endothelial cells causes amplification of the inflammatory response by increased local production of CXCL8 (IL-8), IL-1, IL-6 and TNF- $\alpha[16,20,24]$.

Thus, IL-17A inhibitors produce rapid down-regulation of the psoriasis gene signature, and are associated with a high clinical response in moderate-to-severe plaque psoriasis, which in a way points to the importance of IL-17A in psoriasis pathogenesis [25]. However, the inhibition of IL-17A in cases of psoriatic arthritis and rheumatoid arthritis, has not demonstrated similar efficacy. This suggests that it has a relatively minor role to play in the pathogenesis of inflammatory joint disease or is responsible only in a subset of patients [25].

Many of the newer biologic agents targeting IL-23 and IL-17 have been shown to be safe and highly effective in treating moderate-to-severe plaque psoriasis. However, long-term safety data are still being generated. Unlike TNF- $\alpha$ inhibitors, these agents are highly selective to cause inhibition of targets lower down in the pathogenesis of psoriasis [26].

Because IL-17A is produced locally at the sites of inflammation, targeting it may have the potential for an increased efficacy and much improved safety profile in patients with autoimmune diseases $[27,28]$.

So far, it has been clearly demonstrated that in most of the patients with plaque psoriasis, neutralization of IL-17A is enough for rapid clearance of their psoriatic plaques [27]. Two monoclonal antibodies targeting IL-17A (SEC and IXE), and one antibody against the IL-17 receptor (brodalumab) have been approved for the treatment of moderate-to-severe plaque psoriasis [29].

Ixekizumab is a monoclonal antibody acting specifically against IL-17A [17], and its efficacy and safety in plaque psoriasis has been proved in many phase 3 clinical trials [27, 30, 31].

Similarly, SEC, which is another human IgG1 monoclonal antibody (mAb) that neutralizes IL-17A, has been approved for the treatment of moderate-to-severe plaque psoriasis in adult patients requiring systemic therapy or phototherapy [28].

This 52-week comparative study of the two biologic agents inhibiting IL-17 (IXE vS. SEC) showed that both these agents are effective in our patients of moderate-tosevere psoriasis. However, this study demonstrated that IXE is more efficacious than SEC in attaining PASI 75 and PASI 90 response at week 12; IXE showed significantly higher efficacy than SEC in reducing the signs and symp- 
toms of moderate-to-severe plaque psoriasis (higher reduction in DLQI scores and higher proportion of patients achieving the score of 0 or 1 on the PGA, compared with $\mathrm{SEC})$. The clinical clearance with IXE was not only greater, but also was achieved must faster, and lasted longer after stopping the drug, than with SEC.

Regarding the long-term safety, both the agents exhibited a similar safety profile, including the rates and type of common and serious AEs and laboratory abnormalities. And, no adverse effects leading to withdrawal from the study or deaths were reported.

Farahnik et al. [32] assessed the efficacy and safety profile of the three available anti-IL-17 agents (SEC, IXE and brodalumab) in a phase III clinical trial and found that by week 12 , the proportion of patients achieving PASI 75 was comparable between the different agents (SEC 83\%, IXE 89\%, and brodalumab 85\%). Also, the safety profile of the agents were almost similar, and the most frequently reported AEs were nasopharyngitis, upper respiratory infections, headache, and injection site reactions [32]. For psoriasis, which is a lifelong disease, long-term comparison of the therapeutic agents is important and more relevant clinically [33]. Results of our study offer relatively longer term data on safety and efficacy of different IL-17 inhibitors in a head-to-head comparative trial.

Several studies have compared IL-17 inhibitors with other biologics which are currently licensed for the treatment of psoriasis [27, 28, 34-38]. In one such randomized controlled trial (FIXTURE study) [34] comparing SEC to ustekinumab, SEC showed superior efficacy (79.0\% PASI 90) when compared to ustekinumab (57.6\% PASI 90) at week 16 ( $p<0.0001)$. At week 16, a significantly higher proportion of subjects receiving SEC achieved PASI 100 (44.3\% compared to $28.4 \%$ with ustekinumab) and DLQI score 0/1 (71.9\% compared to $57.4 \%$ with ustekinumab) ( $p$ $<0.0001$ ). And, both drugs had a similar safety profile [34].

Similarly, in another randomized, double-blind study comparing the efficacy and safety of SEC and ustekinumab use over 52 weeks, SEC demonstrated superiority to ustekinumab, with PASI 90 (76\% vs. 61\%) response ( $p<0.0001)$; PASI 100 responses (46\% vs. $36 \%)(p=0.0103)$ and PGA responses of clear/almost clear skin (80\% vs. 65\%) ( $p<0.0001$ ) with a comparable safety profile [35].

Bagel et al., in a head-to-head trial comparing SEC with ustekinumab, reported that SEC was superior to ustekinumab. With a higher proportion of patients achieving PASI 90 (66.5\% vs. 47.9\%) and PGA mod 2011 $0 / 1$ response $(72.3 \%$ vs. $55.4 \%)$ at week $12(p<0.0001)$ in patients who were given SEC [36].

A phase III study comparing IXE with ustekinumab in moderate-to-severe psoriasis showed that at week 24 , IXE achieved PASI 90 in a higher percentage of patients (72.8\% vs. ustekinumab 42.2\%). And, the response rates for PASI 75, PASI 100 and PGA (0 or 1) were significantly higher for IXE than for ustekinumab (adjusted $p<0.05$ ). The safety profiles were consistent with those previously reported for both treatments [37].

A large proportion of psoriasis patients on IXE achieve clear or near clear skin rapidly and sustain the results longer compared to all previously approved drugs for psoriasis. Thus, IXE may cause a paradigm shift in the management of psoriasis, where clear or near clear skin becomes a more accepted and achievable treatment goal [38].

A meta-analysis evaluated the short-term efficacy and safety of biologics targeting IL-17 and IL-23 in the treatment of moderate-to-severe plaque psoriasis. Amongst the twenty-one randomized clinical trials that were included, the results showed that IXE (160 mg $w k 0+80 \mathrm{mg} \mathrm{q2w})$ had the greatest probability of achieving both PASI 75 (RR = 21.32, 95\% CI: 15.48-29.36, $p<0.00001)$ and PASI 90 response $(\mathrm{RR}=59.76,95 \% \mathrm{Cl}$ : $32.41-110.19, p<0.00001)$ at the primary endpoint times. Comparing to the biologics targeting IL-23, the pooled effect size favoured the biological agents targeting IL-17 in terms of PASI 75 (PASI 75: RR $=17.28,95 \% \mathrm{Cl}: 14.51-$ 20.58, $p<0.00001)$ and PASI $90(\mathrm{RR}=37.19,95 \% \mathrm{Cl}$ : 26.91-51.41, $p<0.00001)$. This meta-analysis found that IXE was the most effective short-term treatment [29].

Also, in a review article published recently it has been concluded that IL-17 inhibitors are the preferred agents over other biologics in patients of psoriasis having comorbid conditions like obesity and latent tuberculosis infection $[39,40]$.

As demonstrated in our study, pooled results from individual studies showed that, amongst the various IL inhibitors, IXE achieves higher PASI 75 rates, and similar or higher static PGA 0-1 rates than the other anti-IL-17 and anti-IL-23 agents [39]. The safety profile of IXE is similar to these agents, with nasopharyngitis, upper respiratory infection, headache, arthralgia, and injection-site erythema as the most commonly reported adverse effects [41].

\section{Conclusions}

The anti-IL-17 agents demonstrated a rapid and robust clinical improvement accompanied by a favourable short-term safety profile. The superior efficacy of IXE over SEC observed at week 12 is maintained through week 52 . Thus, IXE seems to be more efficacious in comparison to SEC for the treatment of moderate-to-severe plaque psoriasis, and demonstrates a robust clinical response, noteworthy improvement in patient quality of life, and a satisfactory safety profile. Over 52 weeks, the overall safety of IXE was comparable to that of SEC.

\section{Conflict of interest}

The authors declare no conflict of interest. 


\section{References}

1. Krueger JG, Bowcock A. Psoriasis pathophysiology: current concepts of pathogenesis. Ann Rheum Dis 2005; 64: Suppl 2: ii30-6.

2. Nestle FO, Kaplan DH, Barker J. Psoriasis. N Engl J Med 2009; 361: 496-509.

3. Chon MP, Boehncke WH. Psoriasis. N Engl J Med 2005; 352: 1899-912.

4. Griffiths CEM, Barker JNWN. Pathogenesis and clinical features of psoriasis. Lancet 2007; 370: 263-71.

5. Koo J. Population-based epidemiologic study of psoriasis with emphasis on quality of life assessment. Dermatol Clin 1996; 14: 485-96.

6. Villasenor-Park J, Wheeler D, Grandinetti L. Psoriasis: evolving treatment for a complex disease. Cleve Clin J Med 2012; 79: 413-23.

7. Kim J, Krueger JG. The immunopathogenesis of psoriasis. Dermatol Clin 2015; 33: 13-23.

8. Sharma J, Balakrishnan L, Datta KK, et al. A knowledgebase resource for interleukin-17 family mediated signaling. J Cell Commun Signal 2015; 9: 291-6.

9. Grine L, Dejager L, Libert C, Vandenbroucke RE. An inflammatory triangle in psoriasis: TNF, type I IFNs and IL-17. Cytokine Growth Factor Rev 2015; 26: 25-33.

10. Volpe E, Servant N, Zollinger R, et al. A critical function for transforming growth factor-beta, interleukin 23 and proinflammatory cytokines in driving and modulating human Th17 responses. Nat Immunol 2008; 9: 650-7.

11. Isailovic N, Daigo K, Mantovani A, Selmi C. Interleukin-17 and innate immunity in infections and chronic inflammation. J Autoimmun 2015; 60: 1-11.

12. Malakouti M, Brown GE, Wang E, et al. The role of IL-17 in psoriasis. J Dermatol Treat 2015; 26: 41-4.

13. Ratner M. IL-17-targeting biologics aim to become standard of care in psoriasis. Nat Biotechnol 2015; 33: 3-4.

14. Hanley TL, Yiu ZZ. Role of IL-17 in plaque psoriasis: therapeutic potential of ixekizumab. Ther Clin Risk Manag 2017; 13: 315-23.

15. Zhang X, Angkasekwinai P, Dong C, et al. Structure and function of interleukin-17 family cytokines. Protein Cell 2011; 2: 26-40.

16. Girolomoni G, Mrowietz U, Paul C. Psoriasis: rationale for targeting interleukin-17. Br J Dermatol 2012; 167: 717-24.

17. Liu L, Lu J, Allan BW, et al. Generation and characterization of ixekizumab, a humanized monoclonal antibody that neutralizes interleukin-17A. J Inflamm Res 2016; 9: 39-50.

18. Patel DD, Lee DM, Kolbinger F, Antoni C. Effect of IL-17A blockade with secukinumab in autoimmune diseases. Ann Rheum Dis 2013; 72 Suppl 2: ii116-23.

19. Lin AM, Rubin CJ, Khandpur R, et al. Mast cells and neutrophils release IL-17 through extracellular trap formation in psoriasis. J Immunol 2011; 187: 490-500.

20. Miossec P, Korn T, Kuchroo VK. Interleukin-17 and type 17 helper T cells. N Engl J Med 2009; 361: 888-98.

21. Gaffen SL, Jain R, Garg AV, Cua DJ. The IL-23-IL-17 immune axis: from mechanisms to therapeutic testing. Nat Rev Immunol 2014; 14: 585-600.

22. Gisondi P, Dalle Vedove C, Girolomoni G. Efficacy and safety of secukinumab in chronic plaque psoriasis and psoriatic arthritis therapy. Dermatol Ther 2014; 4: 1-9.

23. Gutowska-Owsiak D, Schaupp AL, Salimi M, et al. IL-17 downregulates filaggrin and affects keratinocyte expression of genes associated with cellular adhesion. Exp Dermatol 2012; 21: 104-10.

24. Fossiez F, Djossou O, Chomarat P, et al. T cell interleukin-17 induces stromal cells to produce proinflammatory and hematopoietic cytokines. J Exp Med 1996; 183: 2593-603.
25. Kirkham BW, Kavanaugh A, Reich K. Interleukin-17A: a unique pathway in immune-mediated diseases: psoriasis, psoriatic arthritis and rheumatoid arthritis. Immunology 2014; 141: 133-42.

26. Campa M, Mansouri B, Warren R, Menter A. A review of biologic therapies targeting il-23 and il-17 for use in moderate-to-severe plaque psoriasis. Dermatol Ther 2016; 6: 1-12.

27. Griffiths CE, Reich K, Lebwohl M, et al. UNCOVER-2 and UNCOVER-3 investigators. Comparison of ixekizumab with etanercept or placebo in moderate-to-severe psoriasis (UNCOVER-2 and UNCOVER-3): results from two phase 3 randomised trials. Lancet 2015; 386: 541-51.

28. Langley RG, Elewski BE, Lebwohl M, et al. ERASURE Study Group; FIXTURE Study Group. Secukinumab in plaque psoriasis - results of two phase 3 trials. N Engl J Med 2014; 371: 326-38.

29. Cui L, Chen R, Subedi S, et al. Efficacy and safety of biologics targeting IL-17 and IL-23 in the treatment of moderate-to-severe plaque psoriasis: a systematic review and meta-analysis of randomized controlled trials. Int Immunopharmacol 2018; 62: 46-58.

30. Gordon KB, Blauvelt A, Papp KA, et al. Phase 3 trials of ixekizumab in moderate-to-severe plaque psoriasis. N Engl J Med 2016; 375: 345-56.

31. Strober B, Leonardi C, Papp KA, et al. Short- and long-term safety outcomes with ixekizumab from 7 clinical trials in psoriasis: etanercept comparisons and integrated data. J Am Acad Dermatol 2017; 76: 432-40.e417.

32. Farahnik B, Beroukhim K, Nakamura M, et al. Anti-IL-17 agents for psoriasis: a review of phase III data. J Drugs Dermatol 2016; 15: 311-6.

33. Paul C, Griffiths CEM, van de Kerkhof PCM, et al. Ixekizumab provides superior efficacy compared with ustekinumab over 52 weeks of treatment: results from IXORA-S, a phase 3 study. J Am Acad Dermatol 2019; 80: 70-9. e3.

34. Thaci D, Blauvelt $A$, Reich K, et al. Secukinumab is superior to ustekinumab in clearing skin of subjects with moderate to severe plaque psoriasis: CLEAR, a randomized controlled trial. J Am Acad Dermatol 2015; 73: 400-9.

35. Blauvelt A, Reich K, Tsai TF, et al. Secukinumab is superior to ustekinumab in clearing skin of subjects with moderate-tosevere plaque psoriasis up to 1 year: Results from the CLEAR study. J Am Acad Dermatol 2017; 76: 60-9.

36. Bagel J, Nia J, Hashim PW, et al. Secukinumab is superior to ustekinumab in clearing skin in patients with moderate to severe plaque psoriasis (16-week CLARITY results). Dermatol Ther 2018; 8: 571-9.

37. Reich K, Pinter A, Lacour JP, et al.; IXORA-S investigators. Comparison of ixekizumab with ustekinumab in moderate-to-severe psoriasis: 24-week results from IXORA-S, a phase III study. $\mathrm{Br}$ J Dermatol 2017; 177: 1014-23.

38. Blegvad C, Skov L, Zachariae C. Ixekizumab for the treatment of psoriasis: an update on new data since first approval. Expert Rev Clin Immunol 2019; 15: 111-21.

39. Kaushik SB, Lebwohl MG. Psoriasis: which therapy for which patient: psoriasis comorbidities and preferred systemic agents. J Am Acad Dermatol 2019; 80: 27-40.

40. Kaushik SB, Lebwohl MG. Psoriasis: which therapy for which patient: focus on special populations and chronic infections. Jm Acad Dermatol 2019; 80: 43-53.

41. Kazemi T, Farahnik B, Koo J, Beroukhim K. Emerging targeted therapies for plaque psoriasis - impact of ixekizumab. Clin Cosmet Invest Dermatol 2017; 10: 133-9. 